\title{
The Relationship Between Apoptotic Activity and Prognostic Factors in Neuroblastomas
}

\author{
Sümeyye EKMEKCi' ${ }^{1}$, Nur OLGUN ${ }^{2}$, Erdener ÖZER ${ }^{1}$ \\ Department of 'Pathology and 'Pediatric Oncology, Dokuz Eylul University, School of Medicine, IZMIR, TURKEY
}

\begin{abstract}
Objective: Prognostic parameters in determining risk groups for treatment in neuroblastoma are cellular differentiation, mitosis karyorrhexis index (MKI), N-myc amplification and age. However, additional prognosticators are still needed because patients can show unpredictable biological behavior. We aimed to study the prognostic significance of apoptotic activity in neuroblastomas.

Material and Method: Thirty-five primary neuroblastoma were evaluated. The data including stage, treatment protocol, metastatic disease, survival, $\mathrm{N}$-myc status, age and prognostic categorization were obtained from the clinical records. The differentiation and MKI were evaluated in hematoxylin and eosin stained slides. Apoptotic activity was assessed by both bcl-2 immunohistochemical staining and the transferase-mediated d-UTP-biotin nick end labeling (TUNEL) method. Data were correlated with established prognostic factors and clinical outcome.

Results: Twenty-five (71.4\%) cases were located in the adrenal. Sixteen cases showed low and 19 high MKI. Thirty-three (\%94) were immunopositive for bcl-2. TUNEL staining was negative in 2 cases. Of the remaining positive 33 cases, 14 had an apoptotic index of $\leq 2 \%, 11$ of $2-4 \%$ and 8 of $\geq 4 \%$. Cases located in the adrenal showed higher scores of bcl-2 positivity than extra-adrenal tumors. There was no statistical significance for both bcl-2 staining and apoptotic index in correlation with cellular differentiation, MKI, N-myc amplification, age and other clinical parameters. Statistical significance was observed between bcl-2 scoring and tumor localization.

Conclusion: According to our results, apoptotic activity is unlikely to be a prognostic parameter in neuroblastomas. Some studies showing significant correlations between clinical outcome and both bcl-2 immunoscoring and apoptotic index, as assessed by the TUNEL method, differences in case numbers and methodology can explain these conflicting results. Larger series and different methodologies are needed to evaluate the prognostic value of apoptotic activity in neuroblastomas.
\end{abstract}

Key Words: Apoptosis, Bcl-2, Neuroblastoma, Prognosis

\section{INTRODUCTION}

Peripheral neuroblastic tumors including neuroblastomas (NBs) are the most common extracranial solid tumors in childhood and infancy that make up approximately 15\% of all tumors within the first four years of childhood $(1,2)$. The common localizations are adrenal medulla (40\%) and sympathetic ganglia of abdominal (25\%), thoracic (15\%), cervical (5\%) and pelvic (5\%) sites (3).

NBs manifest themselves in a variety of clinical conditions ranging from spontaneous regression and maturation to aggressive progression (1). The prognosis depends on the patients' age at the time of diagnosis, presence of metastasis, histological subtypes, and genetic transformations in the tumor tissue (2). Prognostic histological parameters include Schwannian stroma percentage, neuroblastic differentiation and mitosis karyorrhexis index (MKI) (1). The most common genetic anomaly in NB is N-myc amplification and deletion of the short arm of 1st chromosome $(4,5)$.

(Turk Patoloji Derg 2016, 32:99-104)

Received : 07.02.2015 Accepted : 09.12.2015
Although there are established prognostic parameters for NBs, additional prognosticators are still needed because patients can show unpredictable biological behavior.

Apoptosis plays a significant role in tumor formation when it fails to regulate tissue homeostasis and causes an imbalance between cell death and proliferation. Because anticancer therapies aim to kill tumor cells through induction of apoptosis, failure to induce apoptosis may cause tumor resistance to cancer drugs administered in NBs.

Assessment of proteins playing a crucial role in molecular events of apoptosis may enable finding additional prognosticators in NBs. Bcl-2 is an oncoprotein with antiapoptotic and proapoptotic functions and plays a significant role in regulating apoptosis. $\mathrm{Bcl}-2$ protein inhibits apoptosis in two different ways: directly inhibiting pro-caspases or inhibiting the degradation of apoptogenic agents (6).

Correspondence: Erdener ÖZER

Dokuz Eylül Üniversitesi Tip Fakültesi, Patoloji Anabilim Dall,

IZMIR, TURKEY

E-mail: erdener.ozer@deu.edu.tr Phone: +90 2324123407 
Several methods have been used to investigate apoptotic activity. Of these methods, transferase-mediated d-UTPbiotin nick end labeling (TUNEL) method enables demonstrating DNA fragmentation in an in-situ examination. The basic principle of this method is to insert or add marked nucleotides to a single and/or doublestranded DNA breakage (7). In this study, we aimed to evaluate the prognostic significance of apoptotic activity in NBs by using the TUNEL method to assess apoptotic activity and also the immunohistochemical method to evaluate bcl-2 protein expression.

\section{MATERIAL and METHODS}

Patients: The present study comprised 35 NB patients diagnosed and treated at the Dokuz Eylul University Hospital. The clinical parameters such as age, gender, tumor location, clinical tumor stage, presence of distant metastasis, N-myc amplification status, treatment scheme, prognosis and survey were determined from the patients' medical records.

Histopathological examination: The archival hematoxylin and eosin stained sections were reviewed by two pathologists (SE and EO) and the differentiation and MKI of tumor cells were evaluated.

The tumors were subtyped into three groups according to the degree of differentiation: undifferentiated, poorly differentiated, or differentiating NB. If the tumor cells did not show any cytological features indicative of differentiation, such as vesicular appearance of nucleus, development of nucleolus, and enlargement of eosinophilic or amphophilic cytoplasm, a diagnosis of undifferentiated $\mathrm{NB}$ was made, or if less than $5 \%$ of the tumor cells showed features that were indicative of intermediate cells or ganglion cells, a diagnosis of poorly differentiated NB was made. If $5 \%$ or more tumor cells showed features indicative of differentiation, a diagnosis of differentiating NB was made (1).

To evaluate MKI, 5000 tumor cells were counted in 10 or higher power fields depending on the cellularity. Sites that included necrosis, hemorrhage, and autolysis were excluded. The cases were categorized as low MKI if it was less than $2 \%$, moderate if $2-4 \%$ and high if more than $4 \%$ (8).

Bcl-2 immunhistochemistry: The sections were cut from archival tissue blocks and stained with Bcl-2 antibody (Ventana, 1/50 dilution). The immunohistochemical staining was carried out in Ventana BenchMark XT IHC/ISH Staining Module using XT ultraView DAB v3 procedures. Lymph node tissue was used as a positive control. Bcl-2 staining was classified as negative, when positive cells were less than $5 \%$. The positive cases were considered as $1+$ when the percentage of positive cells was $5-75 \%$ and $2+$ when more than $75 \%(9,10)$.

TUNEL staining: The sections were cut from archival tissue blocks, stained using Millipore ApopTag ${ }^{\oplus}$ Plus Peroxidase In Situ Apoptosis Detection Kit S7101 and underwent the routine TUNEL protocol (7). Lymph node tissue was used as a positive control. The apoptotic index (AI) was determined in hot spot areas by counting 1000 cells and calculating the percentage of positive tumor cells. The cases were categorized as low AI if less than 2\%, moderate if 2-4\% and high if more than $4 \%$.

Statistical Analysis: Interobserver variability between two researchers was assessed by using Kappa test. The statistical relation for both Bcl-2 immunoscores and TUNEL AI with morphologic, genetic and clinical parameters was evaluated by performing chi-square test and Mann Whitney U test. A p-value of $<0.05$ was considered statistically significant.

\section{RESULTS}

Overall 35 cases of NB were enrolled into the study. The ages at the time of diagnosis ranged from 1 to 26 years with a median age of 4.1 years. Nine $(25.7 \%)$ patients were younger than 18 months, 19 (54.3\%) between 18 months and 5 years old and $6(17.1 \%)$ older than 5 years old with one case of unknown age (2.9\%). Nineteen (54.3\%) patients were female and $16(45.7 \%)$ male. Twenty-five (71.4\%) cases were located in the adrenal, and $10(28.6 \%)$ in the extra-adrenal organs (Table I).

Four (11.4\%) cases were stage I, two (5.7\%) stage II, seven (20\%) stage III, 15 (42.9\%) stage IV and one (2.9\%) stage IVS. Fourteen (40\%) patients received radiotherapy, whereas $26(74.3 \%)$ cases had chemotherapy. All the patients who received radiotherapy also had chemotherapy. We could not reach the data regarding whether five of the patients had received chemotherapy or not.

The median survival time of the cases was 30 months. Six cases (17.1\%) died of disease and 29 cases (82.8\%) were alive whilst 27 of them had no recurrence and 2 of the alive patients (5.7\%) were being followed up by different centers.

Table II demonstrates the histopathological findings. The Kappa statistics revealed a substantial agreement (Kappa value $=75 \%$ ) between two investigators (EÖ and SE). The final scores in the disagreement cases were decided after two observers reached a consensus after reviewing the cases together. The histological examination revealed 
Table I: Clinical characteristics of the study group

\begin{tabular}{|l|c|}
\hline & (n) (\%) \\
\hline Age & \\
\hline $0-18$ Months & $9(25.7)$ \\
\hline $18-60$ Months & $19(54.3)$ \\
\hline$>60$ Months & $6(17.1)$ \\
\hline Unknown & $1(2.9)$ \\
\hline Gender & $19(54.3)$ \\
\hline Female & $16(45.7)$ \\
\hline Male & $25(71.4)$ \\
\hline Location & $10(28.6)$ \\
\hline Adrenal & \\
\hline Extra-adrenal & $26(74.3)$ \\
\hline Adjuvant Therapy & $14(40)$ \\
\hline Chemotherapy & $14(40)$ \\
\hline Radiotherapy & $5(14.2)$ \\
\hline Chemotherapy + Radiotherapy & \\
\hline Chemotherapy status unknown & $29(82.8)$ \\
\hline Prognosis & $6(17.1)$ \\
\hline Alive & $15(42.9)$ \\
\hline Died & $1(2.9)$ \\
\hline Stage & \\
\hline I & \\
\hline II & \\
\hline III & \\
\hline IV & \\
\hline IVS & \\
\hline Unknown & \\
\hline
\end{tabular}

that $12(34.3 \%)$ cases were undifferentiated, $13(37.1 \%)$ poorly differentiated and $10(28.6 \%)$ differentiated. Sixteen (45.7\%) cases had low MKI and the remaining 19 (54.3\%) cases high MKI. A genetic analysis was conducted in only 22 patients. Of those, six (17.1\%) patients had positive $\mathrm{N}$-myc amplification and 16 (45.7\%) were negative (Table II).

The bcl-2 score was negative in two $(5.7 \%)$ patients, $1(+)$ in six (17.1\%) and 2(+) in 27 (77.1\%) (Figure 1,2). AI was low in $14(40 \%)$ patients, moderate in 11 (31.4\%) and high in $8(22.8 \%)$ (Figure 3,4$)$. TUNEL staining, which was used to measure AI, was negative in two cases (5.7\%) (Table II).

There was no statistically significant difference for bcl-2 staining and TUNEL AI in correlation with age, differentiation, MKI, N-myc amplification, stage, treatment protocol and prognosis. A statistically significant difference
Table II: Histological examination and N-MYC status

\begin{tabular}{|l|c|}
\hline & (n) (\%) \\
\hline Differentiation & $10(28.6)$ \\
\hline Differentiated type & $13(37.1)$ \\
\hline Poorly differentiated type & $12(34.3)$ \\
\hline Undifferentiated type & \\
\hline MKI & $16(45.7)$ \\
\hline Low & $19(54.3)$ \\
\hline High & \\
\hline Bcl-2 score & $2(5.7)$ \\
\hline Negative & $6(17.1)$ \\
\hline $1(+)$ & $27(77.1)$ \\
\hline $2(+)$ & \\
\hline Apoptotic Index & $14(40)$ \\
\hline Low & $11(31.4)$ \\
\hline Moderate & $8(22.8)$ \\
\hline High & \\
\hline N-MYC amplification & $16(45.7)$ \\
\hline Negative & $6(17.1)$ \\
\hline Positive & $13(37.1)$ \\
\hline Unknown & \\
\hline
\end{tabular}

Table III: Apoptotic index and bcl-2 immunohistochemical scores for statistically significant parameters

\begin{tabular}{|l|c|c|}
\hline Tumor localization & High Bcl-2 score $(+2)$ & p value \\
\hline Adrenal $(n=25)$ & $22(88 \%)$ & 0.04 \\
\hline Extra-adrenal $(n=10)$ & $5(50 \%)$ & \\
\hline Sex & Low Apoptotic Index & p value \\
\hline Male $(n=16)$ & $8(50 \%)$ & 0.01 \\
\hline Female $(n=19)$ & $17(89.5 \%)$ & \\
\hline
\end{tabular}

was observed between bcl-2 scoring and tumor localization $(\mathrm{p}=0.04)$. NB cases located in the adrenal showed higher scores of bcl-2 positivity compared to extra-adrenal tumors ( $88 \%$ versus $50 \%$ ). AI assessed by TUNEL was found to be significantly lower in girls $(\mathrm{p}=0.01)$. Low AI was found in $17(89.5 \%)$ female cases in contrast to $8(50 \%)$ males (Table III).

\section{DISCUSSION}

Neuroblastomas are clinically evident and biologically heterogeneous tumors. While advanced stage NBs are likely to metastasize, stage IVS tumors are regressed. In addition some early stage tumors may have a bad prognosis (4). When localized cases (stage I-III) undergo surgical 


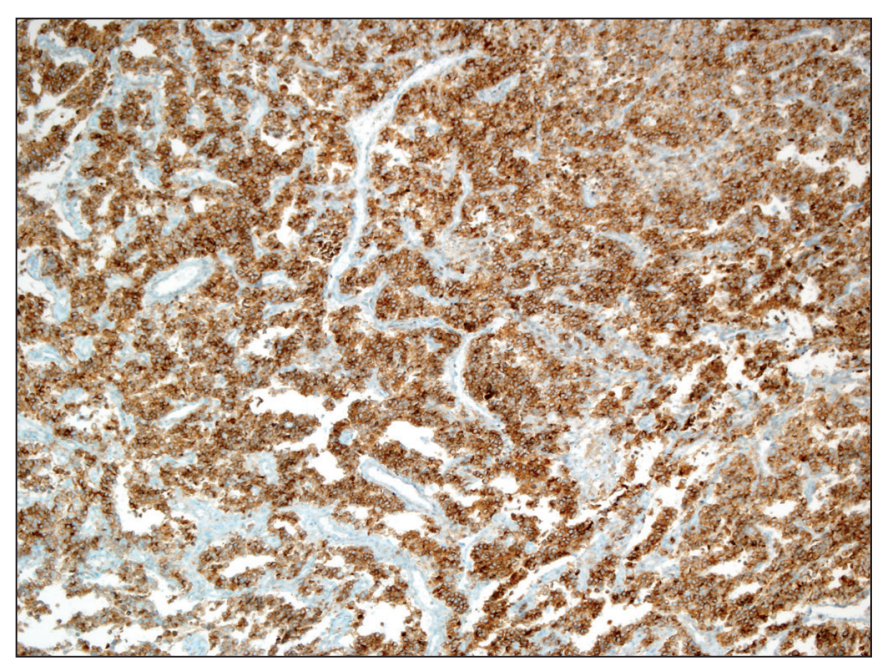

Figure 1: Bcl-2 immunoscoring: $2+$ (bcl-2; x100).

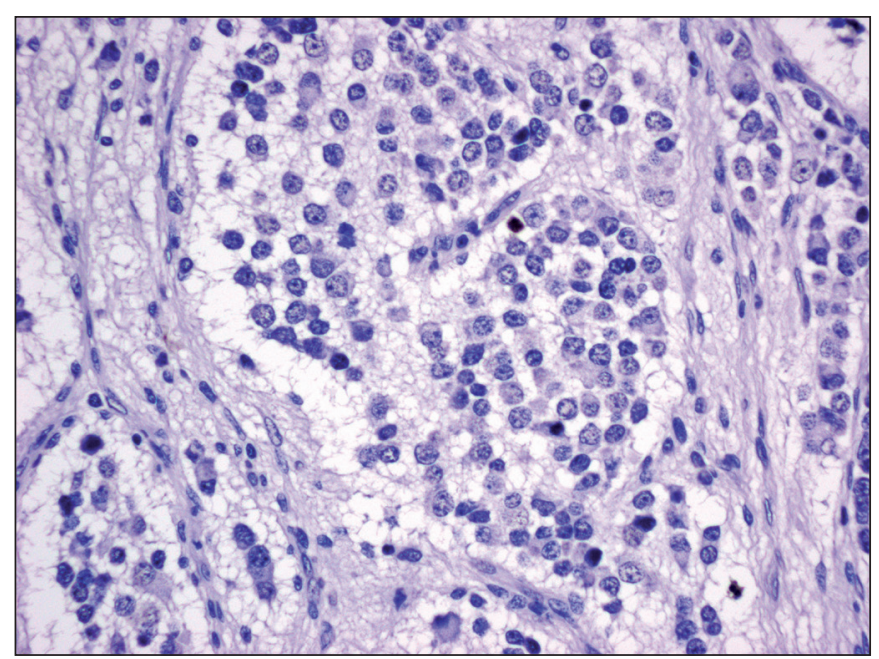

Figure 3: Low apoptotic index (TUNEL staining; $x 400$ ).

resection after adjuvant chemotherapy, the 10-year survival rate may increase to $80 \%$. On the other hand, the 20 -year survival rate is not more than $20 \%$ in metastasized cases (stage IV) even after aggressive chemotherapy. Established histological parameters and biochemical test results cannot explain these biological divergences and the worse prognosis is often explained with molecular test results (11). Although $\mathrm{N}$-myc amplification is characterized by a worse prognosis, $\mathrm{NBs}$ with negative $\mathrm{N}$-myc amplification may have a better clinical outcome (12). Therefore different parameters are still needed to explain the different biological behaviors and define the risk groups for treatment protocols.

Apoptosis is a one of the key mechanisms for tumor development in NBs as in many other tumors. This fact has been already evaluated in several studies in the literature. There are several factors that activate and inhibit apoptosis.

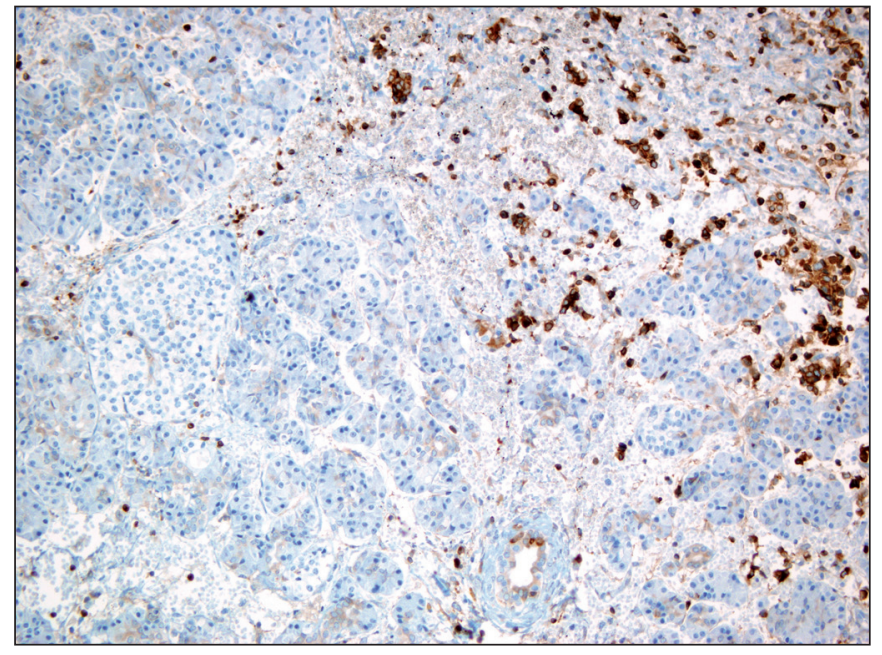

Figure 2: Bcl-2 immunoscoring: 1+ (bcl-2; x200).

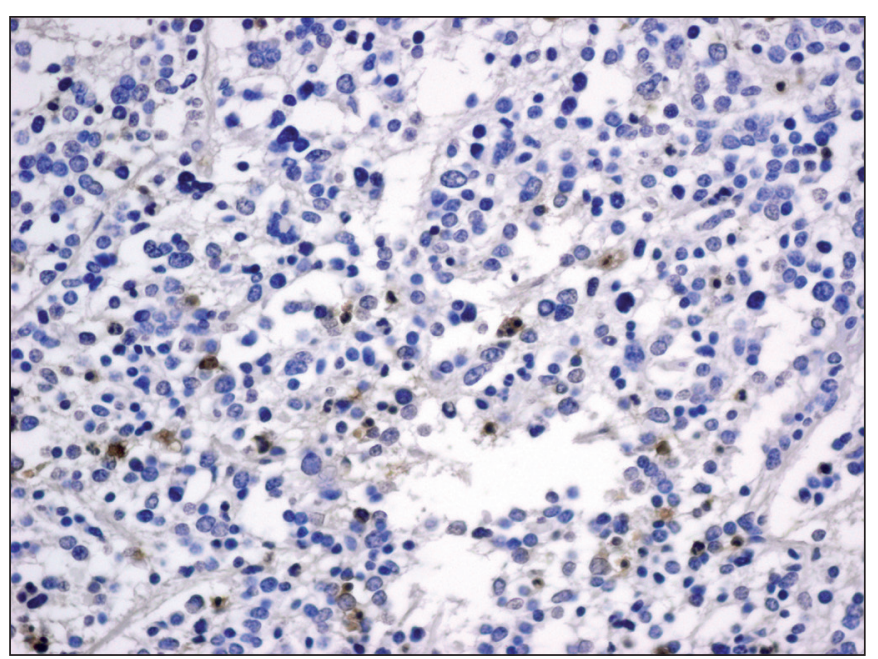

Figure 4: High apoptotic index (TUNEL staining; x400).

The $\mathrm{Bcl}-2$ family is a protooncogene situated outside the mitochondrial membrane and endoplasmic reticulum. Members of the Bcl-2 family serve to sustain cell life and apoptosis of cells. Expression of bcl-2 normally exists in many lymphoid tissues and epithelium cells as well as in the majority of malignancies $(6,9,13)$.

Many investigators have so far studied the Bcl-2 family and the significance of bcl-2 expression on prognosticators of NBs such as histological differentiation, stage, N-myc amplification, prognosis, and treatment. Reed et al. (14) suggested that increased expression of Bcl-2 in NBs prevented apoptosis and prolonged cellular life cycle as well as being able to induce cellular death. In addition, high levels of bcl-2 expression may result in poor response to treatment interacting with many chemotherapeutics with cellular death mechanisms. Castle et al. suggested a 
strong correlation between high bcl-2 expression and both poor differentiation and advanced stage (15). In a study by Hoehner et al., there was a similar correlation between poorly differentiated NB cells and cells with higher immunoscores for bcl-2 immunohistochemistry (16). Similarly Mejia et al. reported that increased Bcl-2 expression was significantly correlated with advanced tumor stage (9).

In our study, 33 of the 35 cases were bcl-2 positive. We found that NBs located in the adrenal had significantly higher immunoscoring for bcl-2 positivity than extraadrenal NBs. There are no data in the literature showing a prognostic difference between adrenal NBs and others with extra-adrenal locations to the best of our knowledge and the correlation between the primary tumor site and biological behavior still remains unexplained. Furthermore, it is interesting to note that cervical NBs are rarely correlated with distant metastasis. In contrast, if the primary site of the tumor is abdomen, $90 \%$ of the patients become metastatic (17).

In contrast to findings in the relevant literature (13-16,18), our study did not reveal any statistically significant relation between bcl-2 expression and established prognostic factors in NBs such as cellular differentiation, tumor stage and $\mathrm{N}$-myc amplification. This contrast may be explained by the different numbers of patients enrolled in the studies and different methodologies used for investigating bcl-2 expression. In addition, cumulative data obtained from studies investigating different proteins and genes involved in the regulation of apoptosis are needed. For example Adida et al. carried out a study to investigate expression of survivin, an antiapoptotic gene, and found a correlation between its expression and progression of the disease (19).

Besides bcl-2 immunohistochemistry, we also assessed AI with the TUNEL method in our study and TUNEL staining was positive in most cases (94.3\%). AI was low in $40 \%$ of the patients, moderate in $31.4 \%$ and high in $22.8 \%$. We did not find any statistically significant relation between $\mathrm{AI}$ and established prognostic factors and other parameters. However, AI was significantly lower in the tumors of female patients. Because of the limited number of cases enrolled in the study, further studies using survival and multivariate analysis are needed to investigate the prognostic significance of apoptotic activity in NB.

In a study by Diensthuber et al., the percentage of TUNEL positivity in NB was similar. Similar to our study, they did not suggest a correlation between AI and prognosis, age, and gender (20). The increased sensitivity of the TUNEL method in comparison with other apoptosis detection methods results from its ability to demonstrate apoptotic cells in micro-sizes that histochemical methods or sophisticated DNA methods commonly fail to demonstrate.

Mejia et al. analyzed apoptotic activity and chromosomal breakages in NBs using in situ methods (9). They conducted their study with 111 cases. The mean age of the patients was 2.1 years and the majority of the patients were male. In contrast, the number of patients in our study was 35 and the mean age 4.1 years. Most of our patients were female. These characteristic differences between the two studies may explain the divergent results.

In summary, based on our results we conclude that apoptotic activity assessed by TUNEL staining and bcl2 immunohistochemistry is unlikely to be a prognostic parameter in NBs. However further studies with larger series and different methodologies are needed to evaluate the prognostic significance of apoptotic activity in NBs and establish it as an evidence-based prognosticator.

\section{REFERENCES}

1. Shimada H, Ambros IM, Dehner LP, Hata J, Joshi VV, Roald B, Stram DO, Gerbing RB, Lukens JN, Matthay KK, Castleberry RP. The International Neuroblastoma Pathology Classification (the Shimada system). Cancer. 1999;86:364-72.

2. Schroeder H, Wacher J, Larsson H, Rosthoej S, Rechnitzer C, Petresen BL, Carlsen NL. Unchanged incidence and increased survival in children with neuroblastoma in Denmark 1981-2000: A population-based study. Br J Cancer. 2009;100:853-7.

3. Joshi VV. Peripheral neuroblastic tumors: Pathologic classification based on recommendations of international neuroblastoma pathology committee (Modification of shimada classification). Pediatr Dev Pathol. 2000;3:184-99.

4. Hiyama E, Hiyama K, Ohtsu K, Yamaoka H, Ichikawa T, Shay JW, Yokoyama T. Telomerase activity in neuroblastoma: Is it a prognostic indicator of clinical behaviour?. Eur J Cancer. 1997;33:1932-6.

5. Schwab M, Westermann F, Hero B, Berthold F. Neuroblastoma: Biology and molecular and chromosomal pathology. Lancet Oncol. 2003;4:472-80.

6. Tsujimoto Y. Role of Bcl-2 family proteins in apoptosis: Apoptosomes or mitochondria? Genes Cells. 1998;3:697-707.

7. Kressel M, Groscurth P. Distinction of apoptotic and necrotic cell death by in situ labelling of fragmented DNA. Cell Tissue Res. 1994;278:549-56.

8. Shimada H, Umehara S, Monobe Y, Hachitanda Y, Nakagawa A, Goto S, Gerbing RB, Stram DO, Lukens JN, Matthay KK. International neuroblastoma pathology classification for prognostic evaluation of patients with peripheral neuroblastic tumors: A report from the Children's Cancer Group. Cancer. 2001;92:2451-61. 
9. Mejia C, Navarro S, Llombart-Bosch A. Apoptosis in peripheral neuroblastic tumors. Immunohistochemical expression of bcl-2 and p53 is related to DNA fragmentation. Histol Histopathol. 2007;22:1365-70

10. Ikeda H, Hirato J, Akami M, Matsuyama S, Suzuki N, Takahashi A, Kuroiwa M. Bcl-2 oncoprotein expression and apoptosis in neuroblastoma. J Pediatr Surg. 1995;30:805-8.

11. Poremba C, Willenbring H, Hero B, Christiansen H, Schäfer KL, Brinkschmidt C, Jürgens $\mathrm{H}$, Böcker W, Dockhorn-Dworniczak B. Telomerase activity distinguishes between neuroblastomas with good and poor prognosis. Ann Oncol. 1999;10:715-21.

12. Hiyama E, Hiyama K, Yokoyama T, Ishii T. Immunohistochemical analysis of $\mathrm{N}$-myc protein expression in neuroblastoma: Correlation with prognosis of patients. J Pediatr Surg. 1991;26:838-43.

13. Negrini M, Silini E, Kozak C, Tsujimoto Y, Croce CM. Molecular analysis of mbcl-2: structure and expression of the murine gene homologous to the human gene involved in follicular lymphoma. Cell.1987;49:455-63.

14. Reed JC, Meister L, Tanaka S, Cuddy M, Yum S, Geyer C, Pleasure D. Differential expression of bcl2 protooncogene in neuroblastoma and other human tumor cell lines of neural origin. Cancer Res. 1991;51:6529-38.
15. Castle VP, Heidelberger KP, Bromberg J, Ou X, Dole M, Nuñez G. Expression of the apoptosis-suppressing protein bcl-2, in neuroblastoma is associated with unfavorable histology and N-myc amplification. Am J Pathol. 1993;143:1543-50.

16. Hoehner JC, Hedborg F, Wiklund HJ, Olsen L, Påhlman S. Cellular death in neuroblastoma: In situ correlation of apoptosis and bcl-2 expression. Int J Cancer. 1995;62:19-24.

17. Cheung NKV, Cohn SL. Neuroblastoma. 1st ed. Berlin, Heidelberg, NewYork:Springer; 2005

18. del Carmen Mejía M, Navarro S, Pellín A, Ruíz A, Castel V, Llombart-Bosch A. Study of proliferation and apoptosis in neuroblastoma. Their relation with other prognostic factors. Arch Med Res. 2002;33:466-72.

19. Adida C, Berrebi D, Peuchmaur M, Reyes-Mugica M, Altieri DC. Anti-apoptosis gene, survivin, and prognosis of neuroblastoma. Lancet. 1998;351:882-883.

20. Diensthuber M1, Potinius M, Rodt T, Stan AC, Welkoborsky HJ, Samii M, Schreyögg J, Lenarz T, Stöver T. Expression of bcl-2 is associated with microvessel density in olfactory neuroblastoma. J Neurooncol. 2008;89:131-9. 